\title{
Inventario y estado de conservación de las charcas ganaderas en la Región de Murcia (SE Península Ibérica)
}

David Verdiell-Cubedo

Departamento de Zoología y Antropología Física, Faculta de Biología, Universidad de Murcia, 30100 Murcia.

\begin{abstract}
Resumen
Correspondencia

D. Verdiell-Cubedo

E-mail: verdiell@um.es

Recibido: 14 marzo 2012

Aceptado: 26 mayo 2012

Publicado on-line: 22 junio 2012

Las charcas ganaderas son sistemas acuáticos temporales asociados a prácticas agropecuarias tradicionales que funcionan como pequeños humedales. Estos sistemas contribuyen de forma significativa a la conservación de la biodiversidad. Trabajos recientes han puesto de manifiesto su elevada importancia para la reproducción de un destacado número de especies de anfibios. Los objetivos de este estudio han sido inventariar y evaluar el estado de conservación de las charcas ganaderas localizadas en la Región de Murcia. Se han inventariado un total de 129 charcas y, a su vez, se ha detectado el uso de estos cuerpos de agua por parte de seis especies de anfibios, tres de ellas incluidas en la Directiva 92/43/CEE: Bufo calamita, Alytes obstetricans y Pelobates cultripes. Un elevado número de charcas habían perdido su funcionalidad o presentaban un estado de conservación deficiente debido al abandono de las tareas de mantenimiento y al exceso de presión ganadera.
\end{abstract}

Palabras clave: Hábitats acuáticos, Charcas temporales, Biodiversidad, Anfibios, Pelobates cultripes, Alytes obstetricans.

\begin{abstract}
Inventory and conservation status of farm ponds in the Region of Murcia (SE Iberian Peninsula)

Farm ponds are temporary aquatic systems related to traditional agricultural practices which act as small wetlands. These systems contribute significantly to the conservation of biodiversity and recent studies have demonstrated their importance for amphibians as breeding sites. The objectives of this study were to provide an inventory of farm ponds and evaluate their conservation status in the Region of Murcia. A total of 129 farm ponds were inventoried and, in turn, a total of six amphibian species were detected, three of them included in the 92/43/CEE Directive: Bufo calamita, Alytes obstetricans and Pelobates cultripes. A large number of ponds were non-operative or showed a poor conservation status mainly due to a neglected maintenance and excessive livestock pressure.
\end{abstract}

Key words: Aquatic habitats, Temporary ponds, Biodiversity, Amphibians, Pelobates cultripes, Alytes obstetricans.

\section{Introducción}

Las charcas ganaderas son sistemas acuáticos temporales, someros, generalmente con una pro- fundidad inferior a un metro, que en la Península Ibérica se encuentran principalmente en zonas de clima mediterráneo árido y semiárido. Su llenado se produce mayormente a partir del agua de lluvia 
por lo que su periodo de inundación o hidroperiodo puede resultar muy irregular intra- e interanualmente (Sancho \& Lacomba 2010) (Fig. 1). En la mayor parte de los casos son sistemas de pequeña entidad, de carácter artificial o seminatural, utilizados y mantenidos tradicionalmente como abrevaderos para el ganado en zonas de secano. La alimentación de las mismas se genera mediante la creación de una zona o cuenca de captación del agua de lluvia, destinada a la generación de escorrentías, inserta en una matriz paisajística forestal o agrícola. Generalmente, la charca se sitúa en una ladera o vaguada de cierta pendiente que facilita la escorrentía y se encuentra delimitada por una mota de tierra o talud que cierra la zona de desagüe, de manera que el agua permanezca retenida. El condicionante para la formación de estos cuerpos de agua es la presencia de sustratos impermeables como las margas y arcillas, que favorecen la escorrentía superficial y resultan de fácil modelado (Ballester 2003).

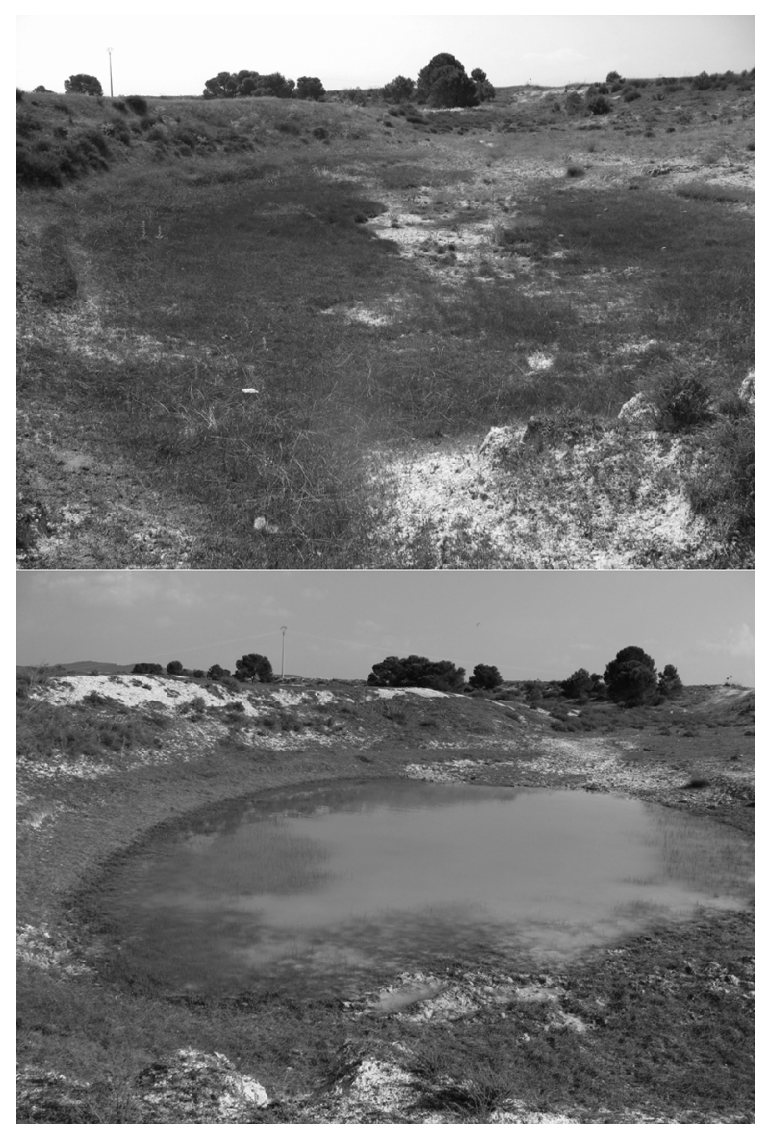

Figura 1. Charca ganadera (C05_Belmonte), situada en la zona de los Llanos del Cagitán, durante la fase seca (arriba) y durante el periodo de inundación (abajo).

Figure 1. Farm pond (C05_Belmonte), located in the Llanos del Cagitán area, during dry period (top) and flood period (down).
Estos ambientes funcionan como pequeños humedales con un elevado grado de naturalidad y poseen una riqueza de especies que las hacen especialmente valiosas para el mantenimiento de la biodiversidad (Cérégrino et al. 2008, Fraga et al. 2010, León et al. 2010, Sancho \& Lacomba 2010; Casas et al. 2011), constituyendo además corredores biológicos que incrementan la conectividad entre otros hábitats de agua dulce (Casas et al. 2012). Por otro lado, son destacables las diversas funciones que pueden desempeñar en zonas áridas y semiáridas, por ejemplo, como bebederos para un gran número de especies de aves y mamíferos (Ballester 2003).

En general, dichos sistemas acuáticos suelen estar asociados a vías pecuarias o sistemas de explotación de ganadería extensiva de carácter local, por lo que poseen un elevado interés cultural e histórico, y son un ejemplo de compatibilidad entre la actividad humana (prácticas agropecuarias tradicionales) y la conservación de la biodiversidad.

En la Región de Murcia este tipo de ambientes constituyen una fracción muy importante de los humedales interiores (hasta un $40 \%$ ), por lo que tienen un papel muy destacado en la conservación de la biodiversidad regional (Ballester 2003). En este sentido, y en relación a las comunidades de anfibios presentes en la Región, trabajos recientes han puesto de manifiesto su elevada importancia para la reproducción de un destacado número de especies. Así, se ha detectado la presencia de Bufo bufo (Linnaeus, 1758), Bufo calamita (Laurenti, 1768), Pelodytes punctatus (Daudin, 1802), Pelophylax perezi (Seoane, 1885), Alytes obstetricans (Laurenti, 1768) y Pelobates cultripes (Cuvier, 1829) (Egea-Serrano et al. 2005, Torralva et al. 2005). De éstas, tres de ellas se encuentran incluidas en el Anexo IV de la Directiva Hábitats 92/43/CEE: B. calamita, A. obstetricans y P. cultripes.

La distribución espacial y delimitación de los sistemas acuáticos en general, y en particular, de las charcas temporales debe ser tenida en cuenta en las estrategias de conservación, puesto que condiciona la distribución de las especies que se desarrollan en ellas (Semlitsch \& Bodie 1998, Revenga et al. 2005). Dado el creciente interés en la conservación de los anfibios y de sus hábitats, el objetivo principal de este estudio ha sido inventariar y evaluar el estado de conservación de las 
charcas ganaderas localizadas en la Región de Murcia, con la finalidad de ofrecer información básica que permita abordar proyectos para su correcta gestión y conservación.

\section{Material y métodos}

El presente trabajo se ha realizado durante los años 2011 y 2012. En una primera fase, y mediante el uso de ortofotos digitales (año 2005) (Visor SIGPAC, Ministerio de Agricultura, Alimentación y Medio Ambiente) y mapas topográficos (escala 1:25.000, Instituto Geográfico Nacional), se localizaron los cuerpos de agua. La búsqueda se centró en áreas geográficas que, gracias a información previa disponible (Ballester 2003, Torralva et al. 2005), reunían las condiciones necesarias para la presencia de este tipo de cuerpos de agua: Altiplano, Llanos del Cagitán, Tierras Altas de Lorca y cabeceras de los ríos Quípar y Pliego.

En una segunda etapa se realizaron muestreos de campo con la finalidad de constatar la presencia y funcionalidad de las charcas, y evaluar su estado de conservación. Las charcas se clasificaron como funcionales si presentaban una lámina de agua o bien, en ausencia de ésta, si se reconocían signos de inundación previa a la fecha de visita (presencia de vegetación palustre, sustrato cuarteado, etc.). Las charcas con signos inequívocos de pérdida de capacidad para retener agua (rotura del talud, colmatación total de la cubeta, etc.) se clasificaron como no funcionales. Las charcas funcionales se evaluaron en relación a su estado de conservación con una puntuación de 1 a 3 (1: deficiente; 2: bueno; 3: óptimo), considerando diversos aspectos relativos a su estructura (tamaño, estructura de la orilla, presencia de vegetación palustre, etc.) y a la presencia de amenazas (presión ganadera elevada, colmatación, presencia de especies exóticas, etc.).

Adicionalmente el medio acuático se prospectó mediante el uso de salabre para detectar la presencia de anfibios (larvas y adultos).

\section{Resultados}

Durante el presente estudio el número de charcas ganaderas inventariadas fue de 129. Las zonas con una mayor abundancia de charcas corresponden a la zona del Altiplano con un total de 41 charcas

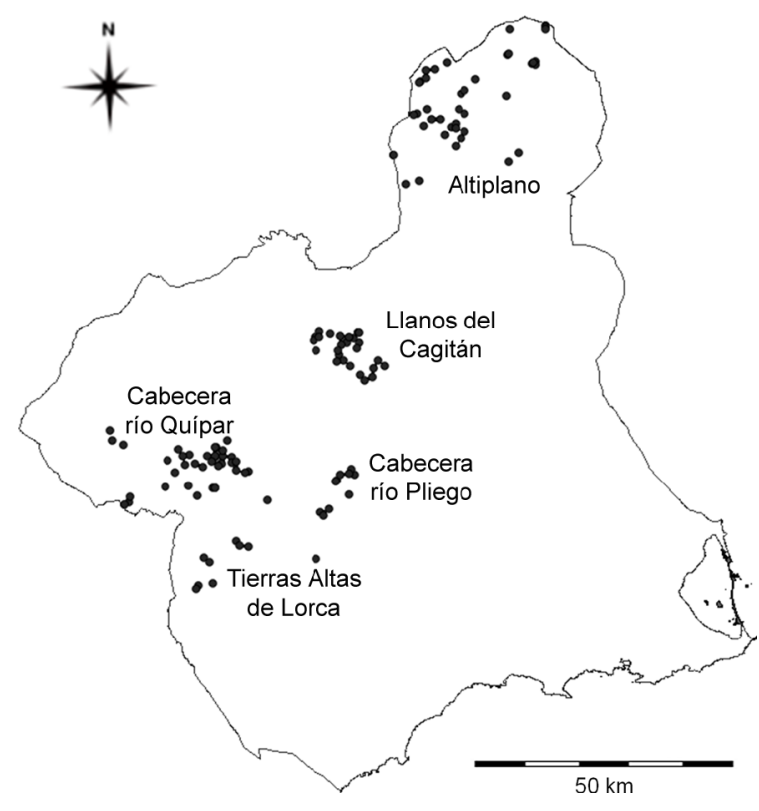

Figura 2. Mapa de distribución de las charcas ganaderas detectadas en la Región de Murcia durante el presente estudio. Se indica la denominación de las zonas prospectadas.

Figure 2. Distribution map of farm ponds detected in the Region of Murcia during this study. It includes the designation of the surveyed areas.

$(31,7 \%)$ y la cabecera del río Quípar (32 charcas, 24,8\%). Les siguen los Llanos del Cagitán con 30 charcas (23,3\%), Tierras Altas de Lorca con 16 $(12,4 \%)$ y cabecera del río Pliego con $10(7,8 \%)$ (Fig. 2 y Apéndice I).

Del total de charcas visitadas $(n=89)$, un $64,0 \%$ (57 charcas) mantenían su funcionalidad, mientras que el resto $(36,0 \%)$, presentaron signos evidentes de pérdida o ausencia de la misma. Por zonas, el Altiplano presentó un mayor número de charcas activas o funcionales, con un total de 18 charcas. A continuación le siguen la cabecera del río Quípar y los Llanos del Cagitán, con un total de 14 y 13 charcas activas, respectivamente. Finalmente, la zona de las Tierras Altas de Lorca presentó 5 charcas activas, mientras que en la cabecera del río Pliego se detectaron 7 charcas funcionales (la totalidad de charcas visitadas en esta zona) (Fig. 3).

En relación al estado de conservación de las charcas, un 36,8\% (21 charcas) presentaron un estado de conservación óptimo, otras 21 charcas $(36,8 \%)$ presentaron un estado de conservación bueno y en el $26,4 \%$ de los casos (15 charcas), se observó un estado de conservación deficiente. La zona del Altiplano acumuló el mayor número de charcas con un estado de conservación deficiente 


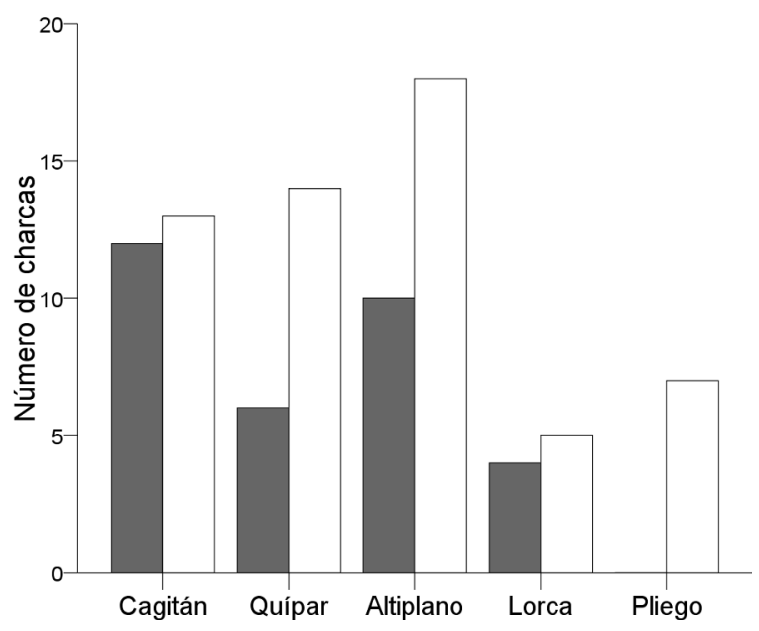

Figura 3. Número de charcas no funcionales (barras grises) y funcionales (barras blancas) en cada una de las zonas de estudio. Figure 3. Number of non-operative farm ponds (grey bars) and operative farm ponds (white bars) in each surveyed area.

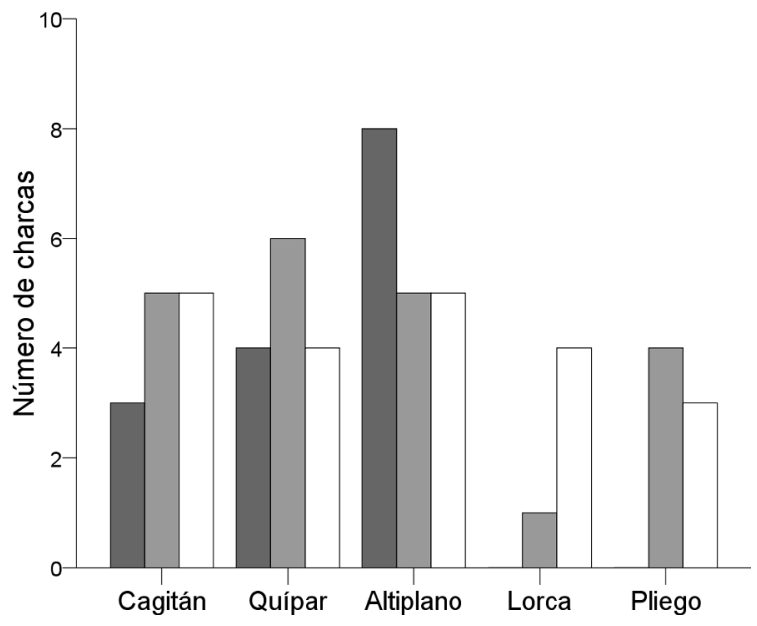

Figura 4. Número de charcas según el estado de conservación para cada una de las zonas de estudio. Barras negras: deficiente; Barras grises: bueno; Barras blancas: óptimo.

Figure 4. Number of farm ponds in terms of conservation status for each surveyed area. Black bars: poor; Grey bars: good; White bars: optimum.

(44,5\% con respecto al total de charcas de esta zona) (Fig. 4). Por el contrario, los Llanos del Cagitán y la cabecera del río Quípar, presentaron un mayor porcentaje de charcas con un estado de conservación óptimo, con un 38,5\% y un 28,6\%, respectivamente. Las charcas distribuidas en Lorca y en la cabecera del río Pliego presentaron, en su mayoría, un estado de conservación óptimo (80\% y 42,8\%, respectivamente) (Fig. 4).

Durante el periodo de estudio fueron detectadas un total de 6 especies de anfibios que utilizaron las charcas ganaderas como hábitat para la reproducción: P. cultripes, B. calamita, B. bufo, A. obstetricans, P. punctatus y P. perezi. Así, se constató la presencia de alguna de estas especies en un total de 35 charcas $(61,4 \%$ respecto a las charcas funcionales). La especie más frecuente fue $P$. cultripes, que estuvo presente en un $31,6 \%$ de las charcas funcionales. La segunda especie más frecuente fue $P$. perezi, localizada en un $28,1 \%$ de las charcas, seguida por $B$. calamita presente en un $24,6 \%$, P. puntactus en un $21,1 \%$ y $A$. obstetricans en un 5,3\%. La reproducción de $B$. bufo fue detectada en una única localidad en la zona de la cabecera del río Pliego.

\section{Discusión}

En el presente trabajo se ha constatado la presencia de, al menos, 129 charcas ganaderas distribuidas ampliamente por el interior de la Región de Murcia. Si comparamos estos resultados con los datos aportados por Ballester (2003), estudio donde se catalogan un total de 35 charcas (si bien no todas corresponden a la categoría de charca ganadera), se observa un evidente incremento en el número de charcas inventariadas. Dicha circunstancia pone de manifiesto la importancia de este tipo de hábitat acuático en el cómputo global de zonas húmedas interiores para la Región.

Aunque el presente trabajo estuvo enfocado al uso de las charcas por parte de la comunidad de anfibios, durante el desarrollo del mismo se pudo comprobar su importancia para otros grupos faunísticos, macrófitos acuáticos y vegetación palustre en general. Así, se observó una gran diversidad de especies de macroinvertebrados, entre los que podemos destacar los branquiópodos Triops cancriformis (Bosc, 1801) y Branchipus spp., e insectos pertenecientes a los órdenes Coleoptera, Heteroptera y Odonata, además del desarrollo de importantes formaciones de vegetación palustre (Juncus spp., Scirpus spp., Phalaris spp., Typha spp.) y macrófitos acuáticos (Potamogeton spp., Chara spp., Zannichellia spp.). En relación a la comunidad de anfibios, cabe destacar la presencia de seis especies de anuros que utilizaron estos cuerpos de agua para la reproducción. Esta cifra representa el $60 \%$ del total de especies de anfibios presentes en la Región de Murcia (Egea-Serrano et al. 2005, Torralva et al. 2005). Como se ha comentado, B. calamita, A. obstetricans y P. cultripes se encuentran incluidas en el Anexo IV de la Directiva Hábitats 92/43/CEE, es decir, son espe- 
cies de interés comunitario que requieren de una protección estricta. Además, conviene destacar el hecho de que $P$. cultripes y $A$. obstetricans presentan un riesgo de extinción medio-elevado en la Región de Murcia (Egea-Serrano et al. 2006). Por tanto, se puede afirmar que las charcas ganaderas representan un hábitat fundamental y prioritario para la conservación de estas especies amenazadas y de la biodiversidad regional en general.

El abandono de las prácticas agropecuarias tradicionales constituye uno de los principales factores de amenaza para la conservación a largo plazo de las charcas ganaderas. El progresivo abandono de la ganadería extensiva y el pastoreo ha supuesto la eliminación de las operaciones de mantenimiento que llevaban a cabo los usuarios, situación que conlleva la pérdida de funcionalidad de la charca y la desaparición definitiva del cuerpo de agua (Ballester 2003, Sancho \& Lacomba 2010). En el caso que nos ocupa, se ha observado que un elevado número de charcas han perdido su funcionalidad o se encuentran en un estado de conservación deficiente, debido mayormente a la colmatación de la cubeta por el arrastre de sedimentos desde la cuenca de captación y a la falta de mantenimiento de las cunetas de escorrentía y taludes de retención. El segundo factor de amenaza en orden de importancia fue el exceso de presión ganadera que, en muchos casos, provoca un incremento importante de la eutrofia de las aguas y la eliminación de la vegetación palustre. En este sentido, el incremento de la intensidad de uso o presión ganadera sobre una determinada charca puede deberse a la pérdida de funcionalidad de otras charcas próximas o localizadas en la misma ruta de tránsito ganadero.

Finalmente, aunque solo con carácter excepcional, se detectó la presencia de la especie exótica Procambarus clarkii (Girard, 1852) en dos charcas localizadas en la zona del Altiplano (A01 Montesinos y A19 Castillo). La presencia de esta especie supone una grave amenaza para las poblaciones de anfibios debido a la acción depredadora que $P$. clarkii ejerce sobre las puestas y larvas (Cruz et al. 2006). En este sentido, sería necesario desarrollar actuaciones encaminadas a erradicar dicha especie en estas charcas y evitar que pueda colonizar charcas próximas.

Por otro lado, en todas las zonas estudiadas cabe destacar la existencia de charcas que, por su buen estado de conservación y la elevada diversi- dad de especies detectada, deberían ser objeto de protección y profundización en la investigación de sus comunidades biológicas. Este es el caso de las charcas C05 Belmonte, C08 Ramel y C12 Carril en los Llanos del Cagitán, las charcas N02 Munueras, N07_Catalán y N16_Reyes en la cabecera del río Quípar, A05_Gaitán, A10_Gamellón y A14_Andaluz en la comarca del Altiplano, L6_Campillo2, L11_Conejos y L14_Gigante en las Tierras Altas de Lorca y las charcas P3_CasasNuevas, P4 AguaBlanca y P7 Bulleros en la cabecera del río Pliego, entre otras (ver Apéndice I). En el contexto de las políticas de gestión y conservación de la naturaleza, competencia de la Administración Regional, deberían adoptarse figuras legales de protección que permitieran su correcta gestión y conservación a largo plazo. En este sentido, debería contemplarse la posibilidad de establecer una figura de protección exclusiva para estos sistemas acuáticos de pequeña entidad, como por ejemplo las Reservas de Fauna Silvestre creadas en la Comunidad Autónoma de Valencia, que han permitido la protección efectiva de un número importante de charcas temporales, junto con la fauna y flora asociadas a las mismas (Lacomba \& Sancho 2009).

Finalmente, sería necesario trabajar en nuevas estrategias complementarias a las llevadas a cabo por las Administraciones en la protección de especies amenazadas y sus hábitats críticos, que conlleven una implicación por parte de otros sectores sociales (Boix et al. 2012). La creación de una Red de Custodia podría contribuir de forma importante a la conservación de las charcas ganaderas mediante la implicación de los propietarios y de voluntarios o ciudadanos interesados en su conservación, ampliando de este modo la extensión de territorio en la protección de estos sistemas acuáticos.

\section{Agradecimientos}

Al Departamento de Zoología y Antropología Física de la Universidad de Murcia y, en particular, al personal de la Línea de Investigación en "Biología y Conservación de Vertebrados Acuáticos", por facilitar la realización del presente trabajo, y a Arianna Alessandro por su inestimable ayuda en el trabajo de campo. También agradezco a Javier Martínez López su imprescindible colaboración para la realización de la Figura 2. Asimismo, mos- 
trar mi gratitud al Dr. Dani Boix por sus comentarios sobre el manuscrito original y su labor en la identificación de la fauna invertebrada.

\section{Referencias}

Ballester R (coord). 2003. Humedales y ramblas de la Región de Murcia. Dirección General del Medio Natural. CARM.

Boix D, Biggs J, Cérégrino R, Hull AP, Kalettka $T$ \& Oertli B: 2012. Pond research and management in Europe: "Small is Beautiful". Hydrobiologia 689: 1-9.

Camacho A, Borja C, Valero-Garcés B, Sahuquillo $M$, Cirujano S, Soria JM, Rico E, De la Hera A, Santamans AC, García de Domingo A, Chicote A \& Gosálvez RU. 2009. 3170* Lagunas y charcas temporales mediterráneas. En: Bases ecológicas preliminares para la conservación de los tipos de hábitat de interés comunitario en España. Madrid: Ministerio de Medio Ambiente, y Medio Rural y Marino.

Casas JJ, Toja J, Bonachela S, Fuentes F, Gallego I, Juan $M$, León $D$, Peñalver $P$, Pérez $C$ \& Sánchez $P$. 2012. Artificial ponds in a Mediterranean region (Andalusia, southern Spain): agricultural and environmental issues. Water and Environment Journal 25: 308-317.

Casas JJ, Toja J, Peñalver P, Juan M, León D, FuentesRodríguez F, Gallego I, Fenoy E, Pérez-Martínez C, Sánchez P, Bonachela S \& Elorrieta MA. 2012. Farm ponds as potential complementary habitats to natural wetlands in a Mediterranean region. Wetlands 32: 161-174.

Cérégrino R, Ruggiero A, Marty P \& Angélibert S. 2008. Biodiversity and distribution patterns of freshwater invertebrates in farm ponds of a south-western French agricultural landscape. Hydrobiologia 597: 43-51.

Cruz MJ, rebelo R \& Crespo EG. 2006. Effects of an introduced crayfish, Procambarus clarkii, on the distribution of south-western Iberian amphibians in their breeding habitats. Ecography 29: 329-338.

Egea-Serrano A, Oliva-Paterna FJ, Miñano $P$, VerdiellCubedo D, de Maya JA, Andreu A, Tejedo M \& Torralva M. 2005. Actualización de la distribución de los anfibios de la Región de Murcia (SE Península Ibérica). Anales de Biología 27: 53-62.

Egea-Serrano A, Oliva-Paterna FJ \& Torralva M. 2006. Amphibians in the Region of Murcia (SE Iberian peninsula): conservation status and priority areas. Animal Biodiversity and Conservation 29: 33-41.

Fraga P, Estaún I \& Cardona E (eds). 2010. Basses temporals mediterrànies. LIFE BASSES: gestió i conservació a Menorca. Consell Insular de Menorca, Maó.

Lacomba I \& Sancho V. 2009. Faunal reserves: a strategy for the protection of small water bodies in Valencia. In International Conference on Mediterranean Temporary Ponds. Proceedings \& Abstracts (Fraga i Arguimbau P, ed). Maó: Consell Insular de Menorca, pp. 445.

León $D$, Peñalver $P$, Casas JJ, Juan $M$, FuentesRodríguez F, Gallego I \& Toja J. 2010. Zooplankton richness in farm ponds of Andalusia (southern Spain). A comparison with natural wetlands. Limnetica 29: 153-162.

Revenga C, Campbell I, Abell R, de Villiers P \& Bryer M. 2005. Prospects for monitoring freshwater ecosystems towards the 2010 targets. Philosophical Transactions of the Royal Society B 360:397-413.

Semlitsch RD \& Bodie JR. 1998. Are small, isolated wetlands expendable? Conservation Biology 12:11291133.

Sancho V \& Lacomba I. 2010. Conservación y Restauración de Puntos de Agua para la Biodiversidad. Colección Manuales Técnicos de Biodiversidad, 2. Generalitat. Conselleria de Medi Ambient, Aigua, Urbanisme i Habitage.

Torralva M, Oliva-Paterna FJ, Egea A, Miñano PA, Verdiell-Cubedo D, de Maya JA \& Andreu A. 2005. Atlas de Distribución de los Anfibios de la Región de Murcia. Dirección General del Medio Natural. CARM. 


\section{Apéndice I}

\begin{tabular}{|c|c|c|c|c|c|c|c|c|c|}
\hline Código charca & UTM (X) & UTM (Y) & $\mathbf{F}$ & EC & Código charca & UTM (X) & UTM (Y) & $\mathbf{F}$ & EC \\
\hline Altiplano & & & & & N11_Casablanca & 578290 & 4206458 & 0 & \\
\hline A01_Montesinos & 631885 & 4264142 & 1 & 1 & N12_LosTellos & 575960 & 4207280 & 0 & \\
\hline A02_Evaristo & 639406 & 4271051 & 1 & 2 & N13_Tartamudo & 575537 & 4209311 & 0 & \\
\hline A03_LaCeja & 642110 & 4268120 & & & N14_LasRevueltas & 597925 & 4205358 & & \\
\hline A04_Bodeguillas & 641255 & 4271193 & & & N15_Floras & 597785 & 4202650 & & \\
\hline A05_Gaitán & 638573 & 4273080 & 1 & 3 & N16_Reyes & 597238 & 4202073 & 1 & 3 \\
\hline A06_Almendros & 637133 & 4278498 & 1 & 2 & N17_Soldado & 587077 & 4203323 & & \\
\hline A07_Almendros2 & 637074 & 4278478 & 0 & & N18_CasaNueva & 594169 & 4202022 & 1 & 1 \\
\hline A08_PeñasBlancas & 638350 & 4280907 & 0 & & N19_Pocicos & 598911 & 4207208 & & \\
\hline A09_AragonaVieja & 643451 & 4269462 & 0 & & N20_Totanero & 599711 & 4202751 & & \\
\hline A10_Gamellón & 644304 & 4270280 & 1 & 3 & N21_Medianiles & 588521 & 4200757 & & \\
\hline A11_Ajiménez & 644374 & 4269315 & 0 & & N22_CasaChico & 591213 & 4198299 & 1 & 2 \\
\hline A12_Quemada & 646074 & 4268670 & 0 & & N23_LomaQuemada & 592895 & 4196197 & 0 & \\
\hline A13_LosCastellanos & 645334 & 4267416 & 0 & & N24_Ventorrillo & 600775 & 4203120 & & \\
\hline A14 Andaluz & 645989 & 4272181 & 1 & 3 & N25 Duque & 594894 & 4204176 & & \\
\hline A15 Beata & 644894 & 4273028 & 0 & & N26 Morenilla & 595916 & 4203084 & & \\
\hline A16 Antonio & 645420 & 4276238 & 0 & & N27 Jarosas & 257 & 5007 & 1 & 2 \\
\hline A17_Cerrillares & 646088 & 4276882 & 0 & & N28_delPozo & 578482 & 4194506 & 1 & 2 \\
\hline A18 Celia & 634313 & 4258162 & 1 & 1 & N29 Marco & 590552 & 2486 & & \\
\hline A19 Castillo & 637012 & 4258671 & 1 & 1 & N30 Rincones & 2447 & 2587 & 1 & 2 \\
\hline A20_Varahonda & 642479 & 4282372 & & & N31_Retamalejo & 546 & 4195926 & 0 & \\
\hline A21_Gateta & 648134 & 4278932 & & & N32_Golilla & 596400 & 4201877 & 1 & 1 \\
\hline A22_Cerro & 654444 & 4275622 & 1 & 1 & Cagitán & & & & \\
\hline A23 Pulpillo & 654717 & 4283846 & & & C01 DelPuerto & 627764 & 4219819 & 0 & \\
\hline A24_Concejil & 654879 & 4284158 & & & C02_Zapata & 623460 & 4222040 & 0 & \\
\hline A25_DonFelipe & 660158 & 4282611 & & & C03 Geromo & 621092 & 4224202 & 1 & 2 \\
\hline A26_Moratillas1 & 659421 & 4282048 & & & C04_Hita & 625432 & 4220288 & 0 & \\
\hline A27 Moratillas2 & 660236 & 4281912 & & & C05 Belmonte & 626272 & 4219205 & 1 & 3 \\
\hline A28_Moratillas3 & 659703 & 4282272 & & & C06_Valenciano & 628802 & 4223083 & & \\
\hline A29_Colorada & 655065 & 4289010 & & & C07_LaParra & 625161 & 4226682 & 0 & \\
\hline A30_CasaRoca & 662189 & 4289678 & 1 & 3 & C08_Ramel & 624080 & 4227527 & 1 & 2 \\
\hline A31_FtePinar & 662169 & 4289297 & 1 & 3 & C09_Mojón & 622610 & 4226637 & 0 & \\
\hline A32 Tobarrillas & 662072 & 4288956 & 1 & 1 & C10 Talones & 624579 & 4225627 & 1 & 1 \\
\hline A33 Portal & 636568 & 4272292 & 1 & 2 & C11 Carpinteros & 383 & 4227723 & 0 & \\
\hline A34 Mayorazqo & 635936 & 4271949 & 1 & 2 & C12 Carril & 133 & 4227140 & 1 & 3 \\
\hline A35 Cenajo & 638 & & 1 & 1 & C13 Cumbres & 084 & 4228740 & 0 & \\
\hline A36 PeñasBlancas2 & 640199 & 4280948 & 1 & 1 & C14 Julia & 619359 & 4228487 & 1 & 2 \\
\hline A37 DelPino & 637888 & 4269709 & & & C15 Saorin & 627939 & 4221658 & 1 & 2 \\
\hline A38 Nava & 654844 & 4262785 & & & C16 FteEscondida & 630199 & 4222053 & 1 & 1 \\
\hline A39_Ardal & 656766 & 4264537 & 1 & 1 & C17_AlfonsoXIII & 621448 & 4228013 & 1 & 3 \\
\hline A40_Grajos & 644245 & 4265779 & 1 & 2 & C18_Guillamón & 624849 & 4228672 & 1 & 1 \\
\hline A41 Ardachos & 653878 & 4261791 & 0 & & C19 Guillamón2 & 624811 & 4228615 & 0 & \\
\hline \multicolumn{5}{|l|}{ Quípar } & C20 Perico & 623172 & 4227672 & 1 & 2 \\
\hline N01_Tarragoya & 591331 & 4204461 & 1 & 1 & C21_Gilico & 617087 & 4227757 & 1 & 3 \\
\hline N02_Munueras & 590207 & 4204097 & 1 & 2 & C22_Collado & 625133 & 4228694 & 0 & \\
\hline N03 Sacejo & 589319 & 4205379 & & & C23 Cortijo & 621507 & 4226132 & & \\
\hline N04_Cabezuelas & 596541 & 4204135 & 1 & 3 & C24_Caputa & 629578 & 421667 & 1 & 3 \\
\hline N05 Peinados & 597953 & 4204169 & 1 & 3 & C25 Domingo & 616542 & 4225178 & & \\
\hline N06 Manzanera & 599842 & 4203864 & 1 & 1 & C26 Calderón & 620871 & 4225157 & 0 & \\
\hline N07_Catalán & 596740 & 4205978 & 1 & 3 & C27_Cagitán & 622048 & 4223048 & 0 & \\
\hline N08 Catalán2 & 596435 & 4205835 & & & C28 Majadas & 620680 & 4222774 & & \\
\hline N09 Gitano & 586639 & 4197910 & 0 & & C29 Calasparra & 616987 & 4228055 & 0 & \\
\hline N10 Campillo1 & 596032 & 4197726 & 1 & 2 & C30 Pozuelo & 622536 & 4225235 & & \\
\hline
\end{tabular}

Apéndice I. Listado de las charcas inventariadas durante el presente estudio. Se indican las coordenadas UTM, funcionalidad (F, 1=funcional; $0=$ no funcional) y estado de conservación (EC, 1=deficiente; $2=$ bueno; $3=$ óptimo).

Appendix I: List of farm ponds inventoried during this study. UTM coordinates, operability (F, 1=operative; $0=$ non-operative) and conservation status (EC, $1=$ poor; $2=$ good; $3=$ optimum) are indicated. 


\begin{tabular}{|c|c|c|c|c|}
\hline Código charca & UTM (X) & UTM (Y) & $\mathbf{F}$ & EC \\
\hline \multicolumn{5}{|l|}{ Lorca } \\
\hline L01 Palomas & 600603 & 4201264 & & \\
\hline L02 Coy & 603004 & 4201057 & 0 & \\
\hline L03 Canadillas & 602373 & 4200883 & 0 & \\
\hline L04_Reñas & 599732 & 4198054 & & \\
\hline L05_Reñas2 & 600090 & 4197952 & & \\
\hline L06 Campillo2 & 596550 & 4197730 & 1 & 3 \\
\hline L07_Ventevacio & 600631 & 4187234 & & \\
\hline L08 PuertoBlanco & 603052 & 4186083 & 0 & \\
\hline L09 Zarcilla & 601229 & 4186272 & & \\
\hline L10 Arenales & 596029 & 4178816 & 1 & 2 \\
\hline L11 Conejos & 593082 & 4178503 & 1 & 3 \\
\hline L12 Peñón & 594228 & 4183912 & 1 & 3 \\
\hline L13_Juncosa & 616499 & 4183732 & & \\
\hline
\end{tabular}

\begin{tabular}{|l|c|c|c|c|}
\hline \multicolumn{1}{|c|}{ Código charca } & UTM (X) & UTM (Y) & F & EC \\
\hline L14_Gigante & 592771 & 4177730 & 1 & 3 \\
\hline L15_Luchena & 595286 & 4183104 & 0 & \\
\hline L16_Muñoz & 606900 & 4195435 & & \\
\hline Pliego & \multicolumn{5}{|c|}{} \\
\hline P01_Almoloya & 617467 & 4193020 & & \\
\hline P02_Rincones & 619177 & 4193600 & 1 & 2 \\
\hline P03_CasasNuevas & 623066 & 4196558 & 1 & 3 \\
\hline P04_AguaBlanca & 620651 & 4199231 & 1 & 3 \\
\hline P05_AguaBlanca2 & 620499 & 4199164 & 1 & 2 \\
\hline P06_Cantina & 618081 & 4192292 & & \\
\hline P07_Bulleros & 624131 & 4200502 & 1 & 2 \\
\hline P08_Bartolo & 623551 & 4201491 & 1 & 2 \\
\hline P09_Camachos & 621329 & 4200369 & 1 & 3 \\
\hline P10_Navarro & 623039 & 4200716 & & \\
\hline
\end{tabular}

Apéndice I (continúa). Listado de las charcas inventariadas durante el presente estudio. Se indican las coordenadas UTM, funcionalidad (F, $1=$ funcional; $0=$ no funcional) y estado de conservación (EC, 1=deficiente; $2=$ bueno; 3=óptimo).

Appendix I (continues): List of farm ponds inventoried during this study. UTM coordinates, operability (F, 1=operative; $0=$ non-operative) and conservation status (EC, $1=$ poor; $2=$ good; $3=$ optimum) are indicated. 